\title{
An Overview of Degradation Phenomena Modeling in Lithium-ion Battery Electrodes
}

\author{
Chien-Fan Chen, Pallab Barai, and Partha P. Mukherjee ${ }^{\mathrm{z}}$ \\ Department of Mechanical Engineering, Texas A\&M University, College Station, TX, USA
}

\author{
Revised manuscript submitted to \\ Current Opinion in Chemical Engineering
}

July 2016

${ }^{z}$ Corresponding author: pmukherjee@tamu.edu 


\begin{abstract}
The formation of solid electrolyte interphase and diffusion induced microcrack in the lithium-ion battery electrodes are predominant degradation mechanisms, which cause capacity fade and cell impedance rise. Physics-based degradation models reveal new insights and allow fundamental understanding of the transport-chemistry-mechanics interactions. In addition, simulation-based diagnostics (e.g. electrochemical impedance spectroscopy, acoustic emission characteristics) can enable virtual probing and interrogation of electrode degradation behavior. This short perspective highlights the recent progress in physics-based degradation modeling and virtual diagnostics in lithium-ion battery electrodes.
\end{abstract}




\section{Introduction}

Life of lithium-ion batteries (LIB) is dictated by the underlying chemo-mechanical degradation mechanisms, such as microcrack formation in the active particles due to the diffusion induced stress generation, [1] and the formation of the solid-electrolyte interphase (SEI) [2-4]. These degradation modes cause capacity fade and impedance rise, thereby affecting the life of LIBs.

During the charging process, the SEI forms as a thin layer $(5 \mathrm{~nm} \sim 300 \mathrm{~nm})$ at the active particle - electrolyte interface.[5] Although the SEI is necessary for preventing undesired reduction and oxidation of the electrode and electrolyte,[6] it also comes with several disadvantages. This chemical degradation mode causes capacity fade due to irreversible consumption of the solvent and active lithium-ions.[7] Moreover, this thin, interfacial layer hinders lithium intercalation, which may increase kinetic resistance.[8]

Mechanical degradation due to diffusion-induced stress (DIS) generation and microcrack formation in LIB electrode active particles also received significant attention in the last few years, as a key degradation mode. $[9,10]$ Microcracks form when the intercalation induced stress in the active particles exceeds the material fracture threshold. Crack formation and propagation from the particle surface may lead to enhanced SEI formation and concurrent loss of cyclable lithium[11]. Pulverization of active particles may further lead to loss of electrical contact [12].

This review aims to provide a brief overview of the modeling studies related to the mechanical (i.e. microcrack formation) and chemical degradation (i.e. SEI formation) mechanisms, and the corresponding influence on the electrochemical properties and performance decay. A discussion on the simulation-driven diagnostics of the degradation behavior, such as electrochemical impedance spectroscopy and acoustic emission, is also included. 


\section{Mathematical Model for the Degradation Phenomena}

\section{Solid Electrolyte Interphase}

The macroscopic models are useful method to predict the SEI formation, and its influence on the capacity lost $[13,14]$ and the increase in internal resistance [15]. Figure 1 shows the SEM image of SEI formation on the graphite particle [2]. To date, different degradation models have been developed to predict the SEI formation and its influence. The model development has been focused on the SEI layer structure $[16,17]$, the thermal effect on the SEI formation $[18,19]$, the influence of SEI of electrochemical properties [20, 21], and the stress generation inside the SEI layer $[22,23]$.

In most of the SEI growth model, the SEI layer is treated as a thin layer. However, the growth of SEI and the morphology of SEI layer vary with position and state-of-charge (SOC). Ploehn et al. [24] proposed a solvent diffusion model, which assumes that a reactive solvent component diffuses through the SEI and undergoes two-electron reduction at the carbon-SEI interface. This model predicted that the SEI thickness growth is proportional to the square root of time. Li et al. [16] studied the formation of SEI in the Graphite/LiFePO 4 batteries, where the SEI layer was assumed to have two layers. The outer SEI layer grows faster than the inner SEI layer, and the formation of SEI is based on electron tunneling. This model also includes the exfoliation of SEI near on the interface between graphite and SEI layer. According to this model, the capacity loss is highly dependent on the SOC due to the electrode potential and the tunneling probability. To study the morphology evolution of the SEI, Guan et al. [17] proposed a phasefield method based model. This model can simulate the SEI structure and morphology evolution. From the predicted SEI structure, the model can predict the lithium-ion diffusivity through the compact, porous, and multilayered structure of SEI layer. 
To include the influence of temperature on the SEI growth, Liu et al [18] and Xie et al [19] developed a 1D electrochemical-thermal coupled model, which includes the growth mechanism of the SEI layer and temperature evolution. The influence of temperature is included by using the Arrhenius equation to determine the temperature-dependent properties.

$$
\Phi=\Phi_{r e f} \exp \left[\frac{E_{\text {act }, \phi}}{R}\left(\frac{1}{T_{r e f}}-\frac{1}{T}\right)\right]
$$

From the results of Liu et al. [18], the SEI layer thickness grows faster under high temperature operation due to the high side reaction rate and the increase of diffusivity. The results from Xie et al [19] shows that high electrolyte salt concentration can stabilize battery performance by compensating the rise of internal resistance due to the consumption of lithiumion in the electrolyte.

Since the presence of SEI layer can hinder the reaction between electrolyte and active material, there is a potential drop across the SEI layer. To capture the potential drop, Phul et al. [20] developed a model, which is able to capture the potential drop according to the diffusion and migration of lithium-ions across the SEI layer. The potential drop from the calculation is fit with the experimental data. From this model, there exists a range of potential drop, which can cause large capacity loss. Besides the prediction of potential drop, Deng et al. [21] proposed a phase field model that takes the non-zero charge density into account, which includes the influence of double layer on the SEI formation. In this model, the SEI formation is treated as a phase transformation process, where the SEI phase is transformed to the SEI phase due to the side electrochemical reaction. From this model, the SEI growth was found to be a diffusionlimited process. This study also found that the growth rate of SEI varied with the initial state-ofcharge of anode. 
According to the experimental study of Mukhopadhyay et al. [25], the SEI formation on a thin-film graphite anode causes reversible and irreversible stresses during the SEI formation. The nominal equi-biaxial stress $\sigma_{n}$ in the combined graphite/SEI film on the quartz substrate can be calculated from the classical Stoney formula.

$$
\sigma_{n}=\left(\frac{1}{6} \frac{E}{\left(1-v_{Q}\right)} \frac{h_{Q}^{2}}{h_{Q}}\right) \kappa
$$

where $\kappa$ is the curvature of the plate, $E_{Q}$ and $v_{Q}$ are the Young's modulus and the Poisson's ratio of the quartz substrate, and $h_{Q}$ and $h_{G}$ are the thicknesses of the quartz and graphite layers.

To simulate the stress generation in the SEI layer, Rejovitzky et al. [22] developed a chemo-mechanical simulation model to predict the SEI growth and the growth stress in the SEI layer. With suitable choices of material parameters, their model can reproduce the experimental results from Mukhopadhyay et al. [25]. According to their results, a large non-uniform compressive hoop stressed is generated in the SEI layer due to both the lithiation/delithiation of the anode particle and the growth of the SEI layer. Besides the stress generation inside the SEI layer, Laresgoiti et al. [23] proposed a model, which predicts the fracture inside the SEI layer. From this model, additional capacity loss during cycling has been found since the fracture in the SEI layer exposes bare active materials to the electrolyte.

\section{Mechanical Damage}

For an electrode, possibility of mechanical degradation depends on the maximum amount of diffusion induced stress at the active particles or the particle/additive (or particle/currentcollector) interface[26]. If the local stress exceeds the fracture threshold at that point, microcracks will evolve and propagate[27]. Figure 2 shows a representative SEM image of microcracks formed in the graphite active particle[28]. Hence, determination of the magnitude of 
stress within the active particle is essential to estimate evolution of mechanical degradation. The pioneering work was conducted by Christensen and Newman, where they looked into the distribution of mechanical stress within carbonaceous anode materials[29]. The presented mathematical model took into consideration the impact of hydrostatic stress and volume expansion during the transport of lithium within spherical active particles. They concluded that carbonaceous anode materials are prone to fracture during high power operations (usually observed in hybrid-electric-vehicles)[29]. This technique has been extended to measure the stress evolution within spherical lithium-manganese-oxide (LMO) cathode materials during two-phase diffusion of lithium[30]. Small volume expansion was considered while estimating transport and mechanical deformation within LMO based active particles. Incorporating formation and movement of dislocations within low volume expansion insertion electrodes, it is possible to reduce the diffusion induced stress experienced during lithiation/delithiation process[31].

The amount of mechanical degradation in intercalation materials is mostly governed by the concentration gradient[27]. Variation in diffusion coefficient with temperature follows the Arrhenius type relation (see Eq. (1)). Reduced diffusivity at lower temperature gives rise to higher concentration gradient, which has the potential to produce enhanced mechanical degradation[32]. Similar to the diffusion coefficient, the fracture threshold parameter and the elastic modulus also vary significantly with temperature. At subzero conditions, graphite anode behaves as a highly brittle material with increased magnitude of elastic modulus, which is prone to rupture. Severe microcracking of the anode has been observed at subzero temperature operation[33]. Ductility of solid materials is also observed to increase at higher temperatures. Hence, negligible mechanical degradation occurs in lithium-ion battery active particles during operation at room temperature[33]. 
Future lithium-ion batteries are expected to offer higher energy density. Increasing the specific capacity of anode has the potential to achieve that goal. However, high capacity anode materials (such as, silicon, tin, germanium) experience 300\% volume expansion during lithiation, which is followed by severe mechanical degradation[34]. Higa and Srinivasan developed a mathematical model to estimate the evolution of mechanical stress within cylindrical silicon active particles under the assumption of elastic deformation[35]. Computational modeling of the two-phase transport of lithium and elastoplastic deformation of silicon active particles has been reported [36]. Concentrated solution theory based lithium transport model coupled with large volume expansion has been developed to study the irreversible changes in atomic structure of silicon during lithiation/delithiation process. This technique permits the creation and destruction of unoccupied silicon lattice sites due to irreversible plastic deformation[37]. Impact of coupled electrical, chemical and mechanical potential on the transport of lithium as well as subsequent mechanical degradation, has been analyzed using a nonlinear finite element based framework[38]. Small strain small displacement based mathematical framework has been developed to study stress generation and delamination experienced by tin micropillars[39] and thin films[26]. They incorporated two-phase diffusion based transport of lithium[26] along with creep deformation of tin active particles[39]. Mathematical models based on large volume expansion and elastoplastic deformation of zinc-oxide nanowires were developed to capture the transport of lithium and subsequent stress generation[40].

Estimation of the magnitude and location of maximum stress gives an idea about whether mechanical degradation is possible within active particles. However, to predict the extent of mechanical damage, evolution of microcracks and propagation of crack fronts must be analyzed. Barai and Mukherjee proposed a lattice spring based computational methodology that is capable 
of estimating initiation, nucleation and propagation of microcracks[27]. Small strain small displacement analysis along with single-phase transport of lithium within graphite electrode was assumed in that study. Figure 3(a) demonstrates the evolution of lithium concentration and subsequent mechanical degradation predicted by this technique. An energy based fracture criteria was adopted to initiate microcracks[27]. A safe design (particle size) and operating (C-rate) regime has been demonstrated in Figure 3(c) where minimum microcrack formation occurs. This lattice-spring based technique was later extended to study the impact of operating temperature on mechanical degradation of graphite based anode active particles[32]. By incorporating nonlinear strain-displacement relations, the lattice spring technique has been modified to model the microcrack formation and propagation within high capacity anode materials, which experience severe volume expansion[41]. The governing expression adopted to solve this problem is:

$$
\int_{t_{V}}{ }_{t}^{t+\Delta t} S_{i j} \delta^{t+\Delta t}{ }_{t} \varepsilon_{i j} d^{t} V-\int_{{ }_{A}}{ }_{t}^{t+\Delta t} F_{i} \delta^{t+\Delta t}{ }_{t} u_{i} d^{t} A={ }^{t+\Delta t} R
$$

Here, ${ }^{t} V$ and ${ }^{t} A$ corresponds to the volume at the previous equilibrium configuration, ${ }^{t+\Delta t} S_{i j}$ is the $2^{\text {nd }}$ Piola-Kirchhoff stress tensor, ${ }^{t+\Delta t}{ }_{t} \varepsilon_{i j}$ is the Green-Lagrange strain tensor, ${ }^{t+\Delta t}{ }_{t} F_{i}$ is the externally applied force, ${ }^{t+\Delta t} u_{i}$ is the displacement and ${ }^{t+\Delta t} R$ is the residual (which is usually considered to be zero). Microcrack evolution in silicon active particles during lithiation process has been demonstrated in Figure 3(b). Phase field based computational methodology has been developed that is capable of simulating simultaneously the propagation of crack fronts as well as flow of electrolyte through the spanning cracks[42]. Cohesive zone based electrochemical model has also been proposed to capture the delamination between binder and active particle during lithiation-delithiation process[43]. 
The mathematical models discussed till now require several mechanical properties as input parameters. Even though some mechanical properties (Young's modulus, shear modulus) of common materials (graphite, silicon, tin) are available in literature, majority of the alloy materials encountered during lithiation are relatively new with unknown properties. Ab-initio calculations using density functional theory (DFT) are useful in estimating the parameters for several new cathode and anode materials[44]. Mechanical properties of lithiated tin and silicon alloy obtained using DFT calculation indicates that, as the amount of lithium increases, Young's modulus decreases along with ductile to brittle transition occurs[45, 46]. Anisotropic and lithium concentration dependent mechanical parameters for lithium-cobalt-oxide have been estimated [47]. Interfacial strengths of silicon/copper and silicon/graphite have been estimated based on first-principle calculations to investigate the mechanical stability of silicon during the lithiation/delithiation process [48, 49]. For materials used in lithium-ion batteries, a comprehensive list of mechanical parameters, as a function SOC, obtained from ab-initio calculations has been reported [44].

\section{Influence of Microstructure}

In most of the macrohomogeneous degradation models, simplifying scenarios such as spherical active particles with uniform size through the electrode are considered. The influence of electrode microstructure heterogeneity is expected to play an important role in the degradation phenomena. Recently, Chen et al. $[15,50]$ computationally studied the effect of microstructural variability on the chemical and mechanical degradation in Lithium-ion battery electrodes. For the study of SEI formation, Chen and Mukherjee [15] developed an electrode microstructure-aware model to observe the spatial variation of SEI formation. The essential concept of this model is schematically shown in Figure 4. This study reveals that the SEI growth rate changes with 
particle size and morphology, and hence the importance of electrode microstructural variation on the degradation mode. In addition, the resistance increase also corroborates well with the Brunauer-Emmett-Teller (BET) specific surface area of graphite active particle suggested by the experimental results of Martin et al. [51]. Chen et al. [50] also integrated the diffusion induced damage model with stochastically reconstructed electrode microstructures. Inside the electrode, the microcrack density was observed to vary with particle size, charge/discharge rate and temperature.

A typical electrode microstructure includes not only the active particles, but also conductive additives and binders. Due to the interaction between individual active particles, microstructure significantly impacts the extent of mechanical degradation observed within lithium-ion batteries. Finite element based analysis in mesoscale level indicates that stresses evolve from small-scale particle features and lithium concentration gradients[52]. Taking into consideration the anisotropic swelling of active particles, it has been demonstrated that maximum stress within electrode occurs due to inter-particle interactions[52]. Incorporation of the impact of hydrostatic stress on lithium transport results in significantly smaller magnitude of concentration gradient, and subsequently reduced mechanical degradation[53]. Using the pseudo 2D porous electrode theory model, increased microcrack evolution has been observed within the active particles located close to the separator[12]. An optimal charging protocol has been developed to minimize the intercalation-induced stress within the active materials [54]. Proper distribution of particles within the electrode has the potential to minimize capacity fade induced by mechanical degradation. 


\section{Simulation-driven Diagnostics}

\section{Electrochemical Impedance Spectroscopy}

As mentioned, the SEI thin layer increases the kinetic resistance for electrochemical reaction. To study the variation of internal resistance due to SEI growth, the electrochemical impedance spectroscopy (EIS) is a powerful tool. Meyer et al. [55] and Huang et al. [56] developed a numerical model, which include the influence of the thickness of SEI layer. The expression of the impedance response is shown below.

$$
Y=\frac{1}{Z}=\frac{1}{\frac{R_{c t, 1}+\frac{R_{p a r t}}{Y_{s}}}{1+j \omega C_{d l, 1}\left(R_{c t, 1}+\frac{R_{p a r t}}{Y_{s}}\right)}+R_{f i l m}+\frac{R_{c t, 2}}{1+j \omega R_{c t, 2} C_{d l, 2}}}+j \omega C_{f i l m}
$$

where $R_{c t, 1}$ and $R_{\text {part }} / Y_{s}$ are the charge transfer and diffusion resistance between the solid phase and SEI layer. $R_{\text {film }}$ and $C_{\text {film }}$ are SEI resistance and capacitance.

This EIS model treats SEI resistance as a layer resistance, and the resistance is a function of film thickness according to the ohm's law. The EIS model proposed by Meyer et al. [55] and Huang et al. [56] did not include the electrochemical reactions, which cannot capture the impedance variation during cycling. To include the growth of SEI during battery cycling, Xie et al. [57] and Chen et al. [15] integrated the EIS model with the electrochemical model. The SEI film growth during cycling is coherently coupled with the electrochemical reaction on the interface between active material and electrolyte. With the coupling, the evolution of impedance during battery cycling is observed. The model developed by Chen et al. [15] further includes the influence of microstructure on the formation of SEI and its corresponding impedance response. The schematic diagram of the model is as shown in Figure 4. The spatial electrochemical properties distribution, such as SEI thickness, was simulated from mesoscale microstructure- 
aware model, and the calculated result was used as inputs to the EIS model. From the results, the electrode resistance contributed by SEI formation is affected by particle morphology, interfacial area, and electrode porosity. Similar to the SEI formation, Chen et al. [50] developed a model, which integrated the diffusion induced damage model and impedance model. This model successfully captured the influence of microcrack on the impedance response. The influence of microcracks is mainly on the solid-phase diffusion resistance and charge transfer resistance.

\section{Acoustic Emission}

Acoustic emission (AE) is a useful nondestructive technique for estimating state of health for lithium-ion batteries. Energy gets released during microcrack formation, chemical reaction with lithium or gas evolution, which travels through the cell as a stress wave (as shown in Figure $5(\mathrm{a}-\mathrm{d}))$. The essence of acoustic emission technique is to detect these travelling waves using ultrasound measurement[58]. Depending on the frequency of detected signal, it is possible to determine the mode of degradation. Low frequency signals $(20-100 \mathrm{kHz})$ are usually associated with gas generation or SEI formation; whereas, high frequency signals $(100-300 \mathrm{kHz})$ are generated because of microcrack nucleation[59]. Acoustic time-of-flight experiment can measure the state-of-charge and state-of-health for any closed battery due to change in density and elastic modulus of cathode and anode materials during charge and discharge process[58]. Acoustic emission technique was first applied on lithium-manganese-oxide cathode active particles to

estimate the mechanical degradation during lithiation-delithiation cycles[60]. Evolution of microcracks within silicon active particles has also been detected using AE techniques[61]. For both LMO and silicon, it has been observed that majority of mechanical degradation occurs within the first few cycles, and microcrack evolution saturates after that[60, 61]. A computational technique was developed based on dynamic lattice spring method to simulate 
crack formation and strain energy release during lithium transport[62]. The following equation has been solved to obtain the displacement $\{U\}$, velocity $\{\dot{U}\}$ and acceleration $\{\ddot{U}\}$ for each of the nodes in the lattice-spring network:

$$
[M]\{\ddot{U}\}+[C]\{\dot{U}\}+[K]\{U\}=\{F\}
$$

Here, $[M]$ signifies the mass matrix, $[C]$ indicates the damping matrix, $[K]$ is the stiffness matrix for the dynamic lattice spring network, and $\{F\}$ corresponds to the force vector due to concentration gradient induced load. As demonstrated in Figure 5(e), under the assumption of brittle fragmentation, saturation in microcrack evolution was observed after the first few cycles. By appropriate calibration and usage of $\mathrm{AE}$, it is possible to use this technique for early detection and diagnosis of degradation and safety concerns in lithium-ion batteries under abuse scenarios.

\section{Conclusion \& Outlook}

This article provides an overview of the modeling of the chemo-mechanical degradation phenomena and highlights some of the recent progress in the simulation-driven degradation diagnostics in lithium-ion battery electrodes. The detrimental effect of SEI and microcrack formation on the capacity fade and impedance rise has been reported from a modeling perspective. The recently reported SEI models provide new insights into the SEI growth and resulting impact on the electrochemical properties and capacity loss. These models reveal that the SEI formation is affected by temperature, state-of-charge, layer morphology, and electrode microstructure. The effects of SEI formation on the potential drop, cell temperature, and stress generation have also been addressed in the recent models. Importance of mechanical degradation in determining the life of lithium-ion batteries has been comprehensively studied using analytical, finite element and lattice spring based models. Two-phase transport of lithium, large 
volume expansion and anisotropic swelling during lithiation can be considered as major factors for severe microcrack formation in active particles. Recent developments in the simulationdriven degradation diagnostics (i.e. electrochemical impedance response and acoustic emission) have been highlighted which are expected to provide virtual probing and prognostics of lithiumion battery life and health.

\section{Acknowledgements}

Financial support from NSF, grant no. 1438431, is gratefully acknowledged. The authors also acknowledge Elsevier, Electrochemical Society, Materials Research Society, and Royal Society of Chemistry for the figures reproduced in this article from the referenced publications of their respective journals. 


\section{References and recommended readings}

1. X. H. Rui, X. X. Zhao, Z. Y. Lu, H. T. Tan, D. H. Sim, H. H. Hng, et al., "Olivine-Type Nanosheets for Lithium-ion Battery Cathodes," Acs Nano, 2013, 7, 5637-5646.

2. P. Verma, P. Maire, and P. Novák, "A Review of the Features and Analyses of the Solid Electrolyte Interphase in Li-Ion Batteries," Electrochimica Acta, 2010, 55, 6332-6341.

3. P. Arora, R. E. White, and M. Doyle, "Capacity fade mechanisms and side reactions in lithium-ion batteries," Journal of the Electrochemical Society, 1998, 145, 3647-3667.

4. J. Vetter, P. Novak, M. R. Wagner, C. Veit, K. C. Moller, J. O. Besenhard, et al., "Ageing mechanisms in lithium-ion batteries," Journal of Power Sources, 2005, 147, 269-281.

5. E. Peled, "The Electrochemical Behavior of Alkali and Alkaline Earth Metals in Nonaqueous Battery Systems-The Solid Electrolyte Interphase Model," Journal of The Electrochemical Society, 1979, 126, 2047-2051.

6. D. Aurbach, B. Markovsky, A. Shechter, Y. Ein - Eli, and H. Cohen, "A Comparative Study of Synthetic Graphite and Li Electrodes in Electrolyte Solutions Based on Ethylene Carbonate - Dimethyl Carbonate Mixtures," Journal of The Electrochemical Society, 1996, 143, 3809-3820.

7. S. Q. Shi, P. Lu, Z. Y. Liu, Y. Qi, L. G. Hector, H. Li, et al., "Direct Calculation of Li-Ion Transport in the Solid Electrolyte Interphase," Journal of the American Chemical Society, 2012, 134, 15476-15487.

8. P. Lu, C. Li, E. W. Schneider, and S. J. Harris, "Chemistry, Impedance, and Morphology Evolution in Solid Electrolyte Interphase Films during Formation in Lithium-ion Batteries," Journal of Physical Chemistry C, 2014, 118, 896-903.

9. Y.-T. Cheng and M. W. Verbrugee, "Diffusion-Induced Stress, Interfacial Charge Transfer, and Criteria for Avoiding Crack Initiation of Electrode Particles.," Journal of The Electrochemical Society, 2010, 157, A508 - A516.

10. S. Golmon, K. Maute, and M. L. Dunn, "Numerical Modeling of ElectrochemicalMechanical Interactions in Lithium Polymer Batteries," Computers and Structures, 2009, 87, 1567 - 1579.

11. J. G. Xu, R. D. Deshpande, J. Pan, Y. T. Cheng, and V. S. Battaglia, "Electrode Side Reactions, Capacity Loss and Mechanical Degradation in Lithium-Ion Batteries," Journal of the Electrochemical Society, 2015, 162, A2026-A2035.

12. P. Barai, K. Smith, C. F. Chen, G. H. Kim, and P. P. Mukherjee, "Reduced Order Modeling of Mechanical Degradation Induced Performance Decay in Lithium-Ion Battery Porous Electrodes," Journal of the Electrochemical Society, 2015, 162, A1751-A1771. 
13. X. K. Lin, J. Park, L. Liu, Y. Lee, A. M. Sastry, and W. Lu, "A Comprehensive Capacity Fade Model and Analysis for Li-Ion Batteries," Journal of the Electrochemical Society, 2013, 160, A1701-A1710.

14. E. Prada, D. Di Domenico, Y. Creff, J. Bernard, V. Sauvant-Moynot, and F. Huet, "A Simplified Electrochemical and Thermal Aging Model of LiFePO4-Graphite Li-ion Batteries: Power and Capacity Fade Simulations," Journal of the Electrochemical Society, 2013, 160, A616-A628.

**15. C.-F. Chen and P. P. Mukherjee, "Probing the Morphological Influence on Solid Electrolyte Interphase and Impedance Response in Intercalation Electrodes," Physical Chemistry Chemical Physics, 2015, 17, 9812-9827.

The authors computationally investigated the electrode microstructure effect on the SEI formation and corresponding impedance response. The interaction among SEI formation, impedance response, and electrode microstructure was demonstrated.

16. D. J. Li, D. Danilov, Z. R. Zhang, H. X. Chen, Y. Yang, and P. H. L. Notten, "Modeling the SEI-Formation on Graphite Electrodes in LiFePO4 Batteries," Journal of the Electrochemical Society, 2015, 162, A858-A869.

**17. P. J. Guan, L. Liu, and X. K. Lin, "Simulation and Experiment on Solid Electrolyte Interphase (SEI) Morphology Evolution and Lithium-Ion Diffusion," Journal of the Electrochemical Society, 2015, 162, A1798-A1808.

Using a phase-field based model, the authors predicted the microstructural and compositional variation of the SEI layer, which showed variation in the effective transport properties through the layer.

*18. L. Liu, J. Park, X. K. Lin, A. M. Sastry, and W. Lu, "A thermal-electrochemical model that gives spatial-dependent growth of solid electrolyte interphase in a Li-ion battery," Journal of Power Sources, 2014, 268, 482-490.

Using the thermal-electrochemical model, the authors reported the thermal behavior of the SEI layer. This model successfully captures the high capacity loss in high-temperature operation.

*19. Y. Y. Xie, J. Y. Li, and C. Yuan, "Multiphysics modeling of lithium-ion battery capacity fading process with solid-electrolyte interphase growth by elementary reaction kinetics," Journal of Power Sources, 2014, 248, 172-179.

Using a thermal-electrochemical model, the authors showed that the rise in internal resistance due to the SEI formation can be alleviated by controlling the lithium concentration in the electrolyte. 
20. S. Phul, A. Deshpande, and B. Krishnamurthy, "A Mathematical model to study the effect of potential drop across the SEI layer on the capacity fading of a lithium-ion battery," Electrochimica Acta, 2015, 164, 281-287.

**21. J. Deng, G. J. Wagner, and R. P. Muller, "Phase Field Modeling of Solid Electrolyte Interface Formation in Lithium-ion Batteries," Journal of The Electrochemical Society, 2013, 160, A487-A496.

In this study, the SEI formation in lithium-ion batteries was predicted based on a phasefield based formalism. This model demonstrates the interaction between the double layer and SEI formation.

22. E. Rejovitzky, C. V. Di Leo, and L. Anand, "A theory and a simulation capability for the growth of a solid electrolyte interphase layer at an anode particle in a Li-ion battery," Journal of the Mechanics and Physics of Solids, 2015, 78, 210-230.

23. I. Laresgoiti, S. Kabitz, M. Ecker, and D. U. Sauer, "Modeling mechanical degradation in lithium-ion batteries during cycling: Solid electrolyte interphase fracture," Journal of Power Sources, 2015, 300, 112-122.

24. H. J. Ploehn, P. Ramadass, and R. E. White, "Solvent diffusion model for aging of lithiumion battery cells," Journal of the Electrochemical Society, 2004, 151, A456-A462.

25. A. Mukhopadhyay, A. Tokranov, X. C. Xiao, and B. W. Sheldon, "Stress development due to surface processes in graphite electrodes for Li-ion batteries: A first report," Electrochimica Acta, 2012, 66, 28-37.

26. C. Wang, Z. Ma, Y. Wang, and C. Lu, "Failure Prediction of High-Capacity Electrode Materials in Lithium-Ion Batteries," Journal of the Electrochemical Society, 2016, 163, A1157 - A1163.

**27. P. Barai and P. P. Mukherjee, "Stochastic Analysis of Diffusion Induced Damage in Lithium-Ion Battery Electrodes," Journal of the Electrochemical Society, 2013, 160, A955-A967.

In this article, the authors developed a lattice spring based computational model to capture the concentration gradient induced microcrack formation and evolution in graphite active particles under disparate operating and cycling conditions.

28. S. J. Harris, R. D. Deshpande, Y. Qi, I. Dutta, and Y.-T. Cheng, "Mesopores Inside Electrode Particles Can Change the Li-Ion Transport Mechanism and DiffusionInduced Stress," Journal of Materials Research, 2010, 25, 1433 - 1440.

**29. J. Christensen and J. Newman, "Stress generation and fracture in lithium insertion materials," Journal of Solid State Electrochemistry, 2006, 10, 293-319. 
Concentration gradient induced stress generation in carbonacous active particles was analyzed. The authors presented a generalized mathematical framework which can be extended for large volume expansion or multiple speies transport in the solid phase.

30. W. H. Woodford, Y. M. Chiang, and W. C. Carter, "Electrochemical Shock in IonIntercalation Materials with Limited Solid-Solubility," Journal of the Electrochemical Society, 2013, 160, A1286-A1292.

31. P. F. Wei, J. Q. Zhou, X. M. Pang, H. X. Liu, K. J. Deng, G. X. Wang, et al., "Effects of dislocation mechanics on diffusion-induced stresses within a spherical insertion particle electrode," Journal of Materials Chemistry A, 2014, 2, 1128-1136.

32. K. An, P. Barai, K. Smith, and P. P. Mukherjee, "Probing the Thermal Implications in Mechanical Degradation of Lithium-Ion Battery Electrodes," Journal of the Electrochemical Society, 2014, 161, A1058-A1070.

33. K. Takahashi and V. Srinivasan, "Examination of Graphite Particle Cracking as a Failure Mode in Lithium-Ion Batteries: A Model-Experimental Study," Journal of the Electrochemical Society, 2015, 162, A635-A645.

34. N. Nitta and G. Yushin, "High-Capacity Anode Materials for Lithium- Ion Batteries: Choice of Elements and Structures for Active Particles," Particle \& Particle Systems Characterization, 2014, 31, 317-336.

35. K. Higa and V. Srinivasan, "Stress and Strain in Silicon Electrode Models," Journal of the Electrochemical Society, 2015, 162, A1111-A1122.

36. L. Chen, F. Fan, L. Hong, J. Chen, Y. Z. Ji, S. L. Zhang, et al., "A Phase-Field Model Coupled with Large Elasto-Plastic Deformation: Application to Lithiated Silicon Electrodes," Journal of the Electrochemical Society, 2014, 161, F3164-F3172.

*37. A. F. Bower, E. Chason, P. R. Guduru, and B. W. Sheldon, "A continuum model of deformation, transport and irreversible changes in atomic structure in amorphous lithium-silicon electrodes," Acta Materialia, 2015, 98, 229-241.

A concentrated solution theory was presented to capture the empty site construction or destruction during transport of lithium in silicon active particles. The authors were able to capture the irreversible volume change in silicon, otherwise reported experimentally.

38. G. Bucci, Y. M. Chiang, and W. C. Carter, "Formulation of the coupled electrochemicalmechanical boundary-value problem, with applications to transport of multiple charged species," Acta Materialia, 2016, 104, 33-51.

39. N. Qaiser, Y. J. Kim, C. S. Hong, and S. M. Han, "Numerical Modeling of FractureResistant Sn Micropillars as Anode for Lithium-ion Batteries," Journal of Physical Chemistry C, 2016, 120, 6953-6962. 
40. Y. F. Zhang, Z. Y. Wang, Y. J. Li, and K. J. Zhao, "Lithiation of ZnO nanowires studied by in-situ transmission electron microscopy and theoretical analysis," Mechanics of Materials, 2015, 91, 313-322.

41. P. Barai and P. P. Mukherjee, "Mechano-Electrochemical Stochastics in High-Capacity Electrodes for Energy Storage," Journal of the Electrochemical Society, 2016, 163, A1120-A1137.

42. M. Klinsmann, D. Rosato, M. Kamlah, and R. M. McMeeking, "Modeling Crack Growth during Li Extraction in Storage Particles Using a Fracture Phase Field Approach," Journal of the Electrochemical Society, 2016, 163, A102-A118.

43. S. Lee, J. Yang, and $\mathrm{W}$. Lu, "Debonding at the interface between active particles and PVDF binder in Li-ion batteries," Extreme Mechanics Letters, 2016, 6, 37 - 44.

**44. Y. Qi, L. G. Hector, C. James, and K. J. Kim, "Lithium Concentration Dependent Elastic Properties of Battery Electrode Materials from First Principles Calculations," Journal of the Electrochemical Society, 2014, 161, F3010-F3018.

Based on first-principle calculations, the authors presented a comprehensive list of mechanical properties, as a function of lithium concentration or state-of-charge, for different electroactive materials in lithium-ion batteries.

45. P. P. Zhang, Z. S. Ma, Y. Wang, Y. L. Zou, W. X. Lei, Y. Pan, et al., "A first principles study of the mechanical properties of Li-Sn alloys," Rsc Advances, 2015, 5, 3602236029 .

46. F. F. Fan, S. Huang, H. Yang, M. Raju, D. Datta, V. B. Shenoy, et al., "Mechanical properties of amorphous LixSi alloys: a reactive force field study," Modelling and Simulation in Materials Science and Engineering, 2013, 21,

47. L. M. Wu and J. Zhang, "Ab initio study of anisotropic mechanical properties of LiCoO2 during lithium intercalation and deintercalation process," Journal of Applied Physics, 2015, 118,

48. M. E. Stournara, Y. Qi, and V. B. Shenoy, "From Ab Initio Calculations to Multiscale Design of Si/C Core-Shell Particles for Li-Ion Anodes," Nano Letters, 2014, 14, 2140 2149.

49. M. E. Stournara, X. C. Xiao, Y. Qi, P. Johari, P. Lu, B. W. Sheldon, et al., "Li Segregation Induces Structure and Strength Changes at the Amorphous Si/Cu Interface," Nano Letters, 2013, 13, 4759-4768. 
**50. C.-F. Chen, P. Barai, and P. P. Mukherjee, "Diffusion Induced Damage and Impedance Response in Lithium-Ion Battery Electrodes," Journal of The Electrochemical Society, 2014, 161, A2138-A2152.

The authors investigated computatinally the electrode microstructure effect on the microcrack formation and corresponding impedance response. The influence of microcrack formation on the impedance rise was examined and estimated.

51. M. Winter, P. Novák, and A. Monnier, "Graphites for Lithium -Ion Cells: The Correlation of the First-Cycle Charge Loss with the Brunauer-Emmett - Teller Surface Area," Journal of The Electrochemical Society, 1998, 145, 428-436.

52. S. A. Roberts, V. E. Brunini, K. N. Long, and A. M. Grillet, "A Framework for ThreeDimensional Mesoscale Modeling of Anisotropic Swelling and Mechanical Deformation in Lithium-Ion Electrodes," Journal of the Electrochemical Society, 2014, 161, F3052-F3059.

53. H. Mendoza, S. A. Roberts, V. E. Brunini, and A. M. Grillet, "Mechanical and Electrochemical Response of a LiCoO2 Cathode using Reconstructed Microstructures," Electrochimica Acta, 2016, 190, 1-15.

54. B. Suthar, P. W. C. Northrop, R. D. Braatz, and V. R. Subramanian, "Optimal Charging Profiles with Minimal Intercalation-Induced Stresses for Lithium-Ion Batteries Using Reformulated Pseudo 2-Dimensional Models," Journal of the Electrochemical Society, 2014, 161, F3144-F3155.

55. J. P. Meyers, M. Doyle, R. M. Darling, and J. Newman, "The Impedance Response of a Porous Electrode Composed of Intercalation Particles," Journal of The Electrochemical Society, 2000, 147, 2930-2940.

56. R. W. J. M. Huang, F. Chung, and E. M. Kelder, "Impedance simulation of a Li-ion battery with porous electrodes and spherical Li+ intercalation particles," Journal of the Electrochemical Society, 2006, 153, A1459-A1465.

57. Y. Xie, J. Li, and C. Yuan, "Mathematical modeling of the electrochemical impedance spectroscopy in lithium-ion battery cycling," Electrochimica Acta, 2014, 127, 266-275.

58. A. G. Hsieh, S. Bhadra, B. J. Hertzberg, P. J. Gjeltema, A. Goy, J. W. Fleischer, et al., "Electrochemical-acoustic time of flight: in operando correlation of physical dynamics with battery charge and health," Energy \& Environmental Science, 2015, 8, 1569-1577.

59. T. Fukushima, S. Kato, N. Kuwata, and J. Kawamura, "In-situ Acoustic Emission Study of Sn Anode in Li ion battery," presented at the ECS Transactions, 2014. 
60. T. Ohzuku, H. Tomura, and K. Sawai, "Monitoring of particle fracture by acoustic emission during charge and discharge of Li/MnO2 cells," Journal of the Electrochemical Society, 1997, 144, 3496-3500.

61. A. Tranchot, A. Etiernble, P. X. Thivel, H. Idrissi, and L. Roue, "In-situ acoustic emission study of Si-based electrodes for Li-ion batteries," Journal of Power Sources, 2015, 279, 259-266.

*62. P. Barai and P. P. Mukherjee, "Mechano-Electrochemical Model for Acoustic Emission Characterization in Intercalation Electrodes," Journal of the Electrochemical Society, 2014, 161, F3123-F3136.

In this article, the authors presented a dynamic lattice spring based computational model capable of estimating the acoustic emission spectra observed during mechanical degradation of electrode active particles. This is expected to enable simulation-driven degradaton diagnostics in lithium-ion batteries. 


\section{List of Figures}

Figure 1. SEM image of a graphite electrode with and without the SEI formation: (a) pristine electrode, (b) electrode after one cycle at $\mathrm{C} / 10$ rate. Adopted from Ref. [2].

Figure 2. SEM image of typical hairline crack propagation in a graphite particle. Adopted from Ref. [28].

Figure 3. (a) Lithium concentration and microcrack formation in a graphite active particle, which experiences small volume expansion, during the delithiation process[27]. (b) Mechanical degradation observed inside silicon active particles during lithiation and subsequent high volume expansion[41]. (c) A phase map between rate of operation and size of active particle showing the regions where minor, a little bit and severe mechanical degradation occurs[27]. Adopted from Refs. [27, 41].

Figure 4. Schematic diagram of the microstructure-aware model, which considers the morphological effect of carbon active material [15]. (a) The implementation of SEI formation model is on the interface between active particle and electrolyte inside the reconstructed electrode microstructure. (b) The reconstructed microstructure, which includes active material, binder, SEI layer, and electrolyte. The calculated state-of-charge and SEI layer thickness are input to the impedance model. (c) The example of impedance response. The radius of first semicircle corresponding to the SEI resistance, the radius of second semi-circle circle corresponding to the charge transfer resistance, and the slope of impedance tail corresponding to the solid-phase diffusion resistance. Adopted from Ref. [15].

Figure 5. (a) Computational reconstruction of an electrode microstructure. (b) Zoomed in view of an electrode active particle spherical in shape. (c) Discretization of the active particle using lattice spring methodology. (d) Demonstration of the axial spring, shear spring and the damper that connects any two adjacent nodes within the lattice spring network. (e) Acoustic emission spectra (black lines) and cumulative strain energy released (red line) during delithiation of a graphite active particle at 4C[62]. Adopted from Ref. [62]. 


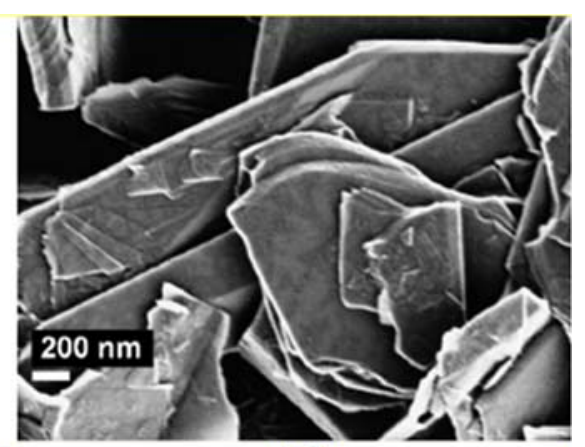

(a)

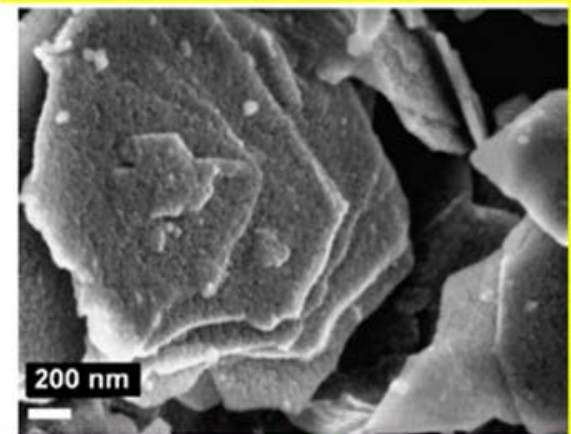

(b)

Figure 1. SEM image of a graphite electrode with and without the SEI formation: (a) pristine electrode, (b) electrode after one cycle at $\mathrm{C} / 10$ rate. Adopted from Ref. [2]. 


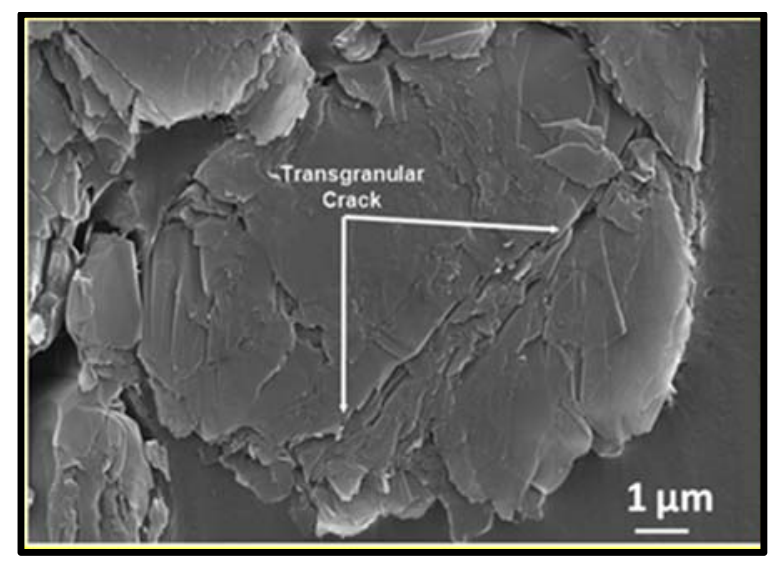

Figure 2. SEM image of typical hairline crack propagation in a graphite particle. Adopted from Ref. [28]. 

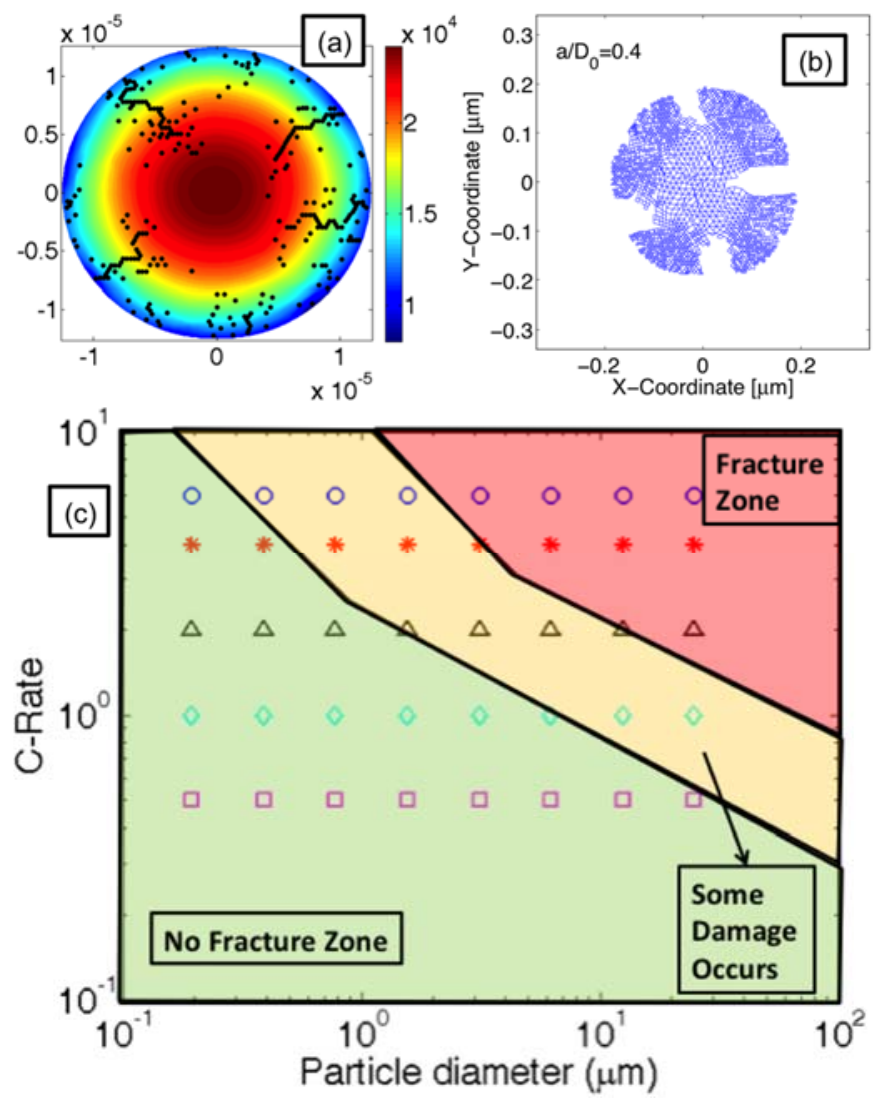

Figure 3. (a) Lithium concentration and microcrack formation in a graphite active particle, which experiences small volume expansion, during the delithiation process[27]. (b) Mechanical degradation observed inside silicon active particles during lithiation and subsequent high volume expansion[41]. (c) A phase map between rate of operation and size of active particle showing the regions where minor, a little bit and severe mechanical degradation occurs[27]. Adopted from Refs. [27, 41]. 


\section{(c)Impedance Model}

\section{(a) Cycling of Active Material}

- Lithiation/Delithiation

- Side Reaction
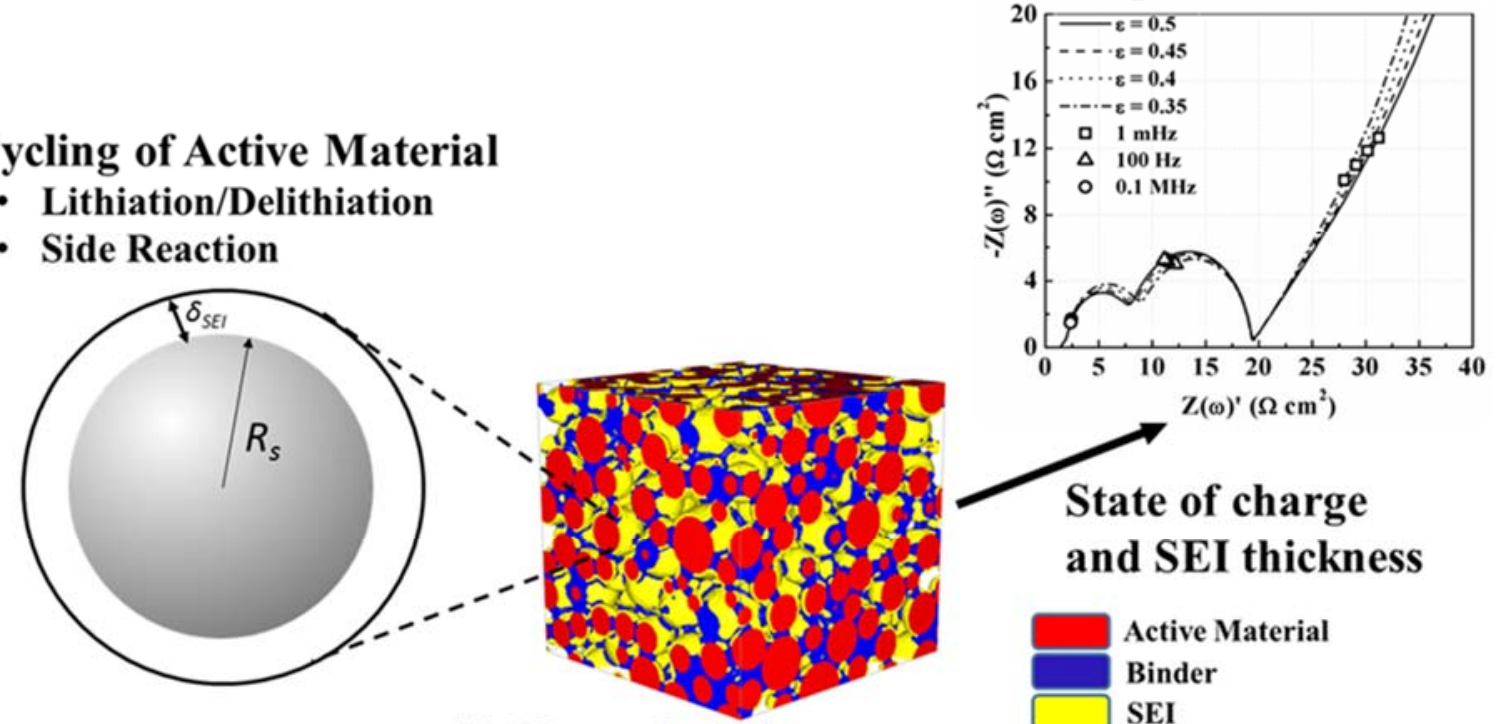

(b)Electrode Microstructure

\section{State of charge and SEI thickness}

Active Material

Binder

SEI

Electrolyte

Figure 4. Schematic diagram of the microstructure-aware model, which considers the morphological effect of carbon active material [15]. (a) The implementation of SEI formation model is on the interface between active particle and electrolyte inside the reconstructed electrode microstructure. (b) The reconstructed microstructure, which includes active material, binder, SEI layer, and electrolyte. The calculated state-of-charge and SEI layer thickness are input to the impedance model. (c) The example of impedance response. The radius of first semicircle corresponding to the SEI resistance, the radius of second semi-circle circle corresponding to the charge transfer resistance, and the slope of impedance tail corresponding to the solid-phase diffusion resistance. Adopted from Ref. [15]. 

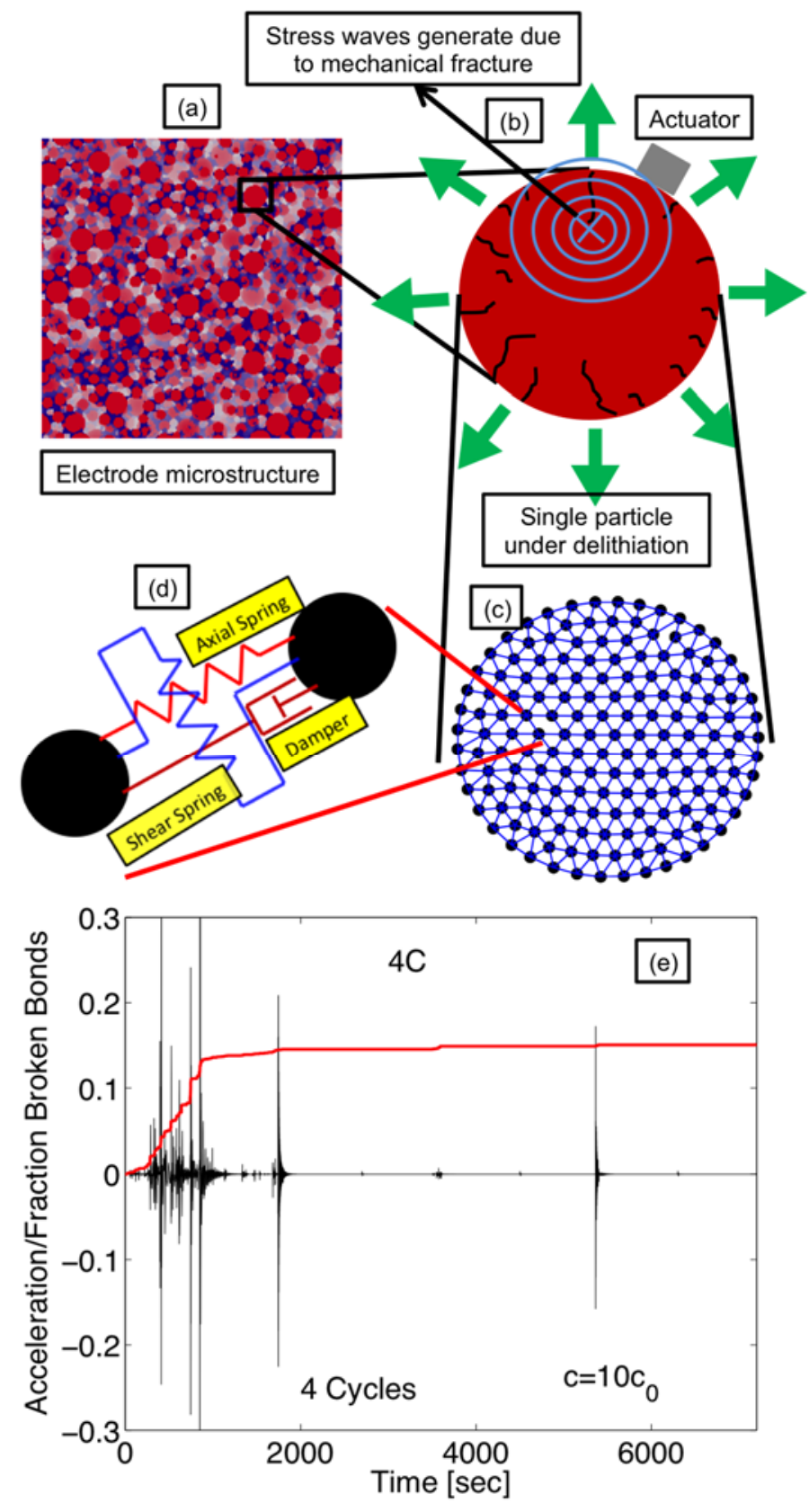

Figure 5. (a) Computational reconstruction of an electrode microstructure. (b) Zoomed in view of an electrode active particle spherical in shape. (c) Discretization of the active particle using lattice spring methodology. (d) Demonstration of the axial spring, shear spring and the damper that connects any two adjacent nodes within the lattice spring network. (e) Acoustic emission spectra (black lines) and cumulative strain energy released (red line) during delithiation of a graphite active particle at 4C[62]. Adopted from Ref. [62]. 